\title{
Dualities in Spin Ladders
}

\author{
Germán Sierra1 ${ }^{10}$ and Miguel A. Martín-Delgado ${ }^{2}$ \\ ${ }^{1}$ Institute of Theoretical Physics, University of California, Santa Barbara, USA \\ ${ }^{2}$ Departamento de Física Teórica I, Universidad Complutense. Madrid, Spain
}

\begin{abstract}
We introduce a set of discrete modular transformations $T_{\ell}, U_{\ell}$ and $S_{\ell}$ in order to study the relationships between the different phases of the Heisenberg ladders obtained with all possible exchange coupling constants. For the 2 legged ladder we show that the $R V B$ phase is invariant under the $S_{\ell}$ transformation, while the Haldane phase is invariant under $U_{\ell}$. These two phases are related by $T_{\ell}$. Moreover there is a "mixed" phase, that is invariant under $T_{\ell}$, and which under $U_{\ell}$ becomes the RVB phase, while under $S_{\ell}$ becomes the Haldane phase. For odd ladders there exists only the $T_{\ell}$ transformation which, for strong coupling, maps the effective antiferromagnetic spin $1 / 2$ chain into the spin $3 / 2$ chain.
\end{abstract}

PACS numbers: 75.10.Jm, 75.50.Ee

In the last two years the concept of duality has played a crucial role in understanding non perturbative aspects of Quantum Field Theoryt and String Theorye. Considering the traditional links between Particle Physics and Statistical Mechanics or Condensed Matter one may wonder wether these latter areas could benefit from the deeper understanding gained in the former ones. In fact, duality ideas have been important in the historical development of Statistical Mechanics, as shown by the Krammers-Wannier duality, order-disorder transformations, etd 3 . In this letter we shall explore the existence of duality symmetries in quantum spin systems defined on a lattice and more particularly on arrays of coupled spin chains known as spin ladders.

Generally speaking a duality transformation is a mapping between two models, or the same model with different parameters, which apparently have different physical properties, but which become in a way equivalent under the transformation. Dual theories usually give complementary descriptions of the same underlying phenomena.

Let us first stablish what we mean by duality in a spin system. We shall consider the Heisenberg Hamiltonian defined on the $d$-dimensional hypercubic lattice $(d \geq 1)$,

$$
H\left(\left\{J_{\mu}\right\}\right)=\sum_{\mu} \sum_{\mathbf{x}} J_{\mu} \mathbf{S}_{\mathbf{x}} \cdot \mathbf{S}_{\mathbf{x}+\mu}
$$

where $\mathbf{S}_{\mathbf{x}}$ is a spin $S$ matrix acting at the position $\mathbf{x}=$ $\left(x_{1}, \ldots, x_{d}\right)$, and $\mu_{1}=(1,0, \ldots, 0), \ldots, \mu_{d}=(0,0, \ldots, 1)$.

We shall define the dual of the Hamiltonian (11) as a Hamiltonian $H_{D}=H\left(\left\{J_{\mu}^{D}\right\}\right)$ characterized by a new set of coupling constants $\left\{J_{\mu}^{D}\right\}$, and such that the low energy spectrum of $H$ and $H_{D}$ is in one to one correspondence. This implies that the free energy and the ground state energy will also be the same for both models.

In the classical limit where the spin $S \gg 1$, the ground state of (11) is given by the classical minima,

$$
\mathbf{S}_{\mathbf{x}}=S \mathbf{n} \prod_{\mu} \epsilon_{\mu}^{\mathbf{x} \cdot \mu}
$$

where $\mathbf{n}$ is a 3 -component unit vector and $\epsilon_{\mu}=-\operatorname{sign} J_{\mu}$. The energy of (2) is given by,

$$
E_{0}^{\text {class }}=-S^{2} \sum_{\mu} \sum_{\mathbf{x}}\left|J_{\mu}\right|
$$

The signs of the exchange coupling constants, $\epsilon_{\mu}$, determine the type of order parameter which characterizes the ground state. Thus $\epsilon_{\mu}=1$ or -1 correspond to ferromagnetic $(F)$ or antiferromagnetic $(A)$ order in the direction $\mu$ of the lattice. Altogether there are $2^{d}$ possible classical vacua which we denote, for $d=2$, by the sequence,

$$
A A, A F, F A, F F
$$

The energies of the classical g.s. and the excitations are independent of the type of vacua (4). All the classical Heisenberg models are equivalent. However the quantum corrections drive them into very different quantum vacua. Only the pure ferromagnetic system (i.e. $\left.J_{\mu}<0, \forall \mu\right)$, survives the quantum fluctuations, but the non-ferromagnetic systems change deeply their structure. The purpose of this letter is to show the relations existing between the different vacua by means of a certain type of duality transformations.

At this point it is useful to make an analogy between $2 \mathrm{~d}$ spin systems and fermions living on a $2 \mathrm{~d}$ torus a fermion on a torus one has to specify the boundary conditions along the $a$ and $b$ cycles. They can be periodic $(P)$ or antiperiodic $(A)$. This gives rise to 4 possible spin structures, labeled as $A A, A P, P A$ and $P P$, which mix under the action of the modular transformations $T, U$ and $S$ as follows

$$
\begin{aligned}
& T: A A \leftrightarrow A P, P A, P P \\
& U: A A \leftrightarrow P A, A P, P P \\
& S: A P \leftrightarrow P A, A A, P P
\end{aligned}
$$

Observe that the spin structure $P P$ is left invariant under the action of the modular group. The fermion determinant with the boundary conditions $A A, A P, P A$ turns 
out to be given by Jacobi $\vartheta$ functions, which transform among themselves under the modular group as described by (5). The fermion determinant for PP boundary conditions is zero due to the existence of a zero mode.

In the case of $2 \mathrm{~d}$ spin systems the role of the cycles $a$ and $b$ is played by the directions $\mu_{1}=(1,0)$ and $\mu_{2}=$ $(0,1)$. The analog of the spin structure is given by the (anti)ferromagnetic nature of the coupling $J_{\mu}$ along the directions $\mu_{1,2}$. Finally, a modular transformation is a redefinition of the unit cell of the lattice. In the case of spin ladders the above analogies can be collected in the following dictionary,

Torus Lattice $\leftrightarrow$ Spin Ladder

$$
\begin{aligned}
& a-\text { cycle } \leftrightarrow \text { legs } \\
& b-\text { cycle } \leftrightarrow \text { rungs }
\end{aligned}
$$

Antiperiodic BC $\leftrightarrow$ Antiferromagnetic Coup.

Periodic BC $\leftrightarrow$ Ferromagnetic Coup.

Modular Transf. $\leftrightarrow$ Bond Moving Transf.

These correspondences have an analogue in $d>2$. On a 2 legged ladder, 2-ladder for short, we shall define three transformations $T_{\ell}, U_{\ell}$ and $S_{\ell}$ as follows. The $T_{\ell}$ transformation consists in the shift by one lattice spacing of one leg respect to the other one (see fig.1). The $U_{\ell}$ transformation consist in the permutation of the two sites of the even rungs, while leaving invariant the odd ones (see fig.2). Finally the $S_{\ell}$ transformation is defined by the equation $S_{\ell}=T_{\ell} U_{\ell} T_{\ell}$, and has the effect of converting all the vertical bonds (rungs) into horizontal ones (legs), while half of the horizontal bonds become vertical bonds and the other half become diagonal bonds of length $\sqrt{5}$. We remark that $T_{\ell}, U_{\ell}$ and $S_{\ell}$ do not generate the standard modular group.

Using these definitions one can see that the classical vacua $A A, A F, F A$ and $F F$, get mixed under the action of $T_{\ell}, U_{\ell}, S_{\ell}$ in the form described by (5), with the replacement: (anti)periodic $\leftrightarrow$ (anti)ferromagnetic. The term "bond moving" in (16) refers to a transformation introduced by Migdal and Kadanoff in the study of the Ising model with RG methods 6 . Just like a fermion on a lattice with non periodic BC's can be regarded as essentially the same object, we conjecture that the 2-ladder, and more generally the ladders with an even number of legs, with "magnetic structures", $A A, A F, F A$, belong to the same universality class and are related through dual transformations. This conjecture implies in particular the equivalence between the RVB state ( $A A$ couplings) and the Haldane state ( $A F$ couplings) of the 2-ladder studied by various authors, $\mathrm{O}$, but it also suggests new equivalences which have not been studied so far. We shall confine ourselves in this letter to the case of the spin $1 / 2$ ladder with 2 legs, trying to prove the above conjecture using perturbative and field theoretical techniques. At the end we shall briefly consider the case of odd ladders.

Let us start with a toy ladder.

\section{A $2 \times 2$ cluster}

The simplest 2-ladder has 4 spins coupled by the Hamiltonian,

$$
H=J_{a}\left(\mathbf{S}_{1} \cdot \mathbf{S}_{2}+\mathbf{S}_{3} \cdot \mathbf{S}_{4}\right)+J_{b}\left(\mathbf{S}_{1} \cdot \mathbf{S}_{4}+\mathbf{S}_{3} \cdot \mathbf{S}_{2}\right)
$$

The ground state of the non-ferromagnetic Hamiltonians (i.e. $\epsilon_{a, b} \neq 1$ ) is a singlet and therefore can be written as the linear combination

$$
\begin{gathered}
|\psi\rangle=\tau|a\rangle+|b\rangle \\
|a\rangle=(12)(34), \quad|b\rangle=(14)(32)
\end{gathered}
$$

where $(i j)$ denotes the singlet valence bond state constructed out from the spin 1/2's located at the sites $i$ and $j$. The transformations $S_{\ell}, T_{\ell}$ and $U_{\ell}$ of figure 1 become for the toy ladder elementary transpositions,

$$
T_{\ell}: 3 \leftrightarrow 4, U_{\ell}: 2 \leftrightarrow 3, S_{\ell}: 2 \leftrightarrow 4
$$

The action of $T_{\ell}, U_{\ell}, S_{\ell}$ on the states (8) can be easily derived from (8) and (9). They are given in table 1 .

\begin{tabular}{|c|c|c|c|}
\hline & $T_{\ell}$ & $U_{\ell}$ & $S_{\ell}$ \\
\hline$|a\rangle$ & $-|a\rangle$ & $|a\rangle-|b\rangle$ & $|b\rangle$ \\
$|b\rangle$ & $-|a\rangle+|b\rangle$ & $-|b\rangle$ & $|a\rangle$ \\
\hline
\end{tabular}

Table 1.

The "modular transformations" induced on the "modular parameter" $\tau$ that follow from table 1 are,

$$
T_{\ell}: \tau \rightarrow-(\tau+1), U_{\ell}: \tau \rightarrow-\tau /(\tau+1), S_{\ell}: \tau \rightarrow 1 / \tau
$$

which are similar but not identical to the standard modular transformations of the torus.

The ground state energy of (7) is,

$$
E=-\frac{1}{2}\left(J_{a}+J_{b}\right)-\sqrt{J_{a}^{2}+J_{b}^{2}-J_{a} J_{b}}
$$

corresponding to a value of $\tau$ given by,

$$
\tau=-1+\frac{J_{a}}{J_{b}}-\epsilon_{b} \sqrt{1-\frac{J_{a}}{J_{b}}+\left(\frac{J_{a}}{J_{b}}\right)^{2}}
$$

The values of $\tau$ obtained by changing the signs and strengths of the exchange coupling constants $J_{a, b}$, cover the whole real axis as described in table 2 .

\begin{tabular}{|c|c|c|c|c|}
\hline$\left(\epsilon_{a}, \epsilon_{b}\right)$ & $A F$ & $F F$ & $F A$ & $A A$ \\
\hline$\tau$ & $(-\infty,-2)$ & $(-2,-1 / 2)$ & $\left(-\frac{1}{2}, 0\right)$ & $(0, \infty)$ \\
\hline
\end{tabular}

Table 2 .

We have included the case $F F$ which corresponds to an excited state, since the g.s. is a spin 2 multiplet. The $S_{\ell}$ transformation (10) leaves invariant the $A A$ and $F F$ domains, while interchanges the regions $A F$ and $F A$. $S_{\ell}$-duality is an exact symmetry of the Hamiltonian (7). Actually, $\tau=1$ is a fixed point of $S_{\ell}$. The $T_{\ell}$ and $U_{\ell}$ transformations are approximate symmetries in the sense that the Hamiltonian (7) is not mapped into a similar 
one with a redefinition of $J_{a, b}$. However one can see that $\tau=-1 / 2$ is a fixed point of $T_{\ell}$, while the $A A$ region $\tau>1$ is mapped under $T_{\ell}$ into the $A F$ region $\tau<-2$. Similarly $\tau=-2$ is a fixed point of $U_{\ell}$, while the $A A$ region $0<\tau<1$ is mapped under $U_{\ell}$ into the $F A$ region $-1 / 2<\tau<0$. All these shows that eqs. (5) hold with some caveats for the $2 \times 2$ cluster.

Within each domain, $A A, A F$ and $F A$, we shall choose a representative state $|\tau\rangle$ with the property of being invariant under one of the dual transformations. The state $\tau=1$ can be called a $R V B$ state since it describes the resonance between two vertical and horizontal bonds. The state $\tau=-2$ is a Haldane like state $(H A L)$ in the sense that it is obtained upon forming the spin 1 state along the rungs, which then couple to form a singlet. Finally $\tau=-1 / 2$ is a mixed state $(M I X)$, corresponding to ferromagnetic chains coupled antiferromagnetically. Moreover, each of the states $|R V B\rangle,|H A L\rangle$ and $|M I X\rangle$ gets transformed into one another by the action of $T_{\ell}, U_{\ell}, S_{\ell}$.

The results are summarized in table 3 .

\begin{tabular}{|c|c|c|c|c|c|}
\hline$\left(J_{a}, J_{b}\right)$ & State & $\tau$ & $S_{\ell}$ & $U_{\ell}$ & $T_{\ell}$ \\
\hline$A A$ & $R V B$ & 1 & $R V B$ & $M I X$ & $H A L$ \\
$A F$ & $H A L$ & -2 & $M I X$ & $H A L$ & $R V B$ \\
$F A$ & $M I X$ & $-1 / 2$ & $H A L$ & $R V B$ & $M I X$ \\
\hline
\end{tabular}

Table 3 .

For 2-ladders with a large number of rungs we can still make sense of the transformation properties collected in table 3 . In that case $|R V B\rangle$ denotes the ground state of a ladder with $A A$ couplings, etc.

The rest of the letter will be devoted to show the validity of table 3 .

The Weak Coupling Regime: $T_{\ell}$-duality

If the two legs are weakly coupled (i.e. $\left|J_{a} / J_{b}\right|>>$ $1)$, the $T_{\ell}$-duality becomes a manifest symmetry of the effective low energy theory.

For $J_{a}>0$ we can use bosonization techniques to show this fact. Indeed the effective ladder Hamiltonian can be written in the bosonized model as 10 ,

$$
\begin{gathered}
H=H_{\mathrm{WZW}}+\lambda_{1}\left(\mathbf{J}_{L} \cdot \mathbf{J}_{R}+\hat{\mathbf{J}}_{L} \cdot \hat{\mathbf{J}}_{R}\right) \\
+\lambda_{2}\left(\mathbf{J}_{L} \cdot \hat{\mathbf{J}}_{R}+\hat{\mathbf{J}}_{L} \cdot \mathbf{J}_{R}\right)+\lambda_{3} \operatorname{Tr}(g \sigma) \operatorname{Tr}(\hat{g} \sigma)+\lambda_{4} \operatorname{Tr} g \operatorname{Tr} \hat{g}
\end{gathered}
$$

where $g$ and $\mathbf{J}$ (resp. $\hat{g}$ and $\hat{\mathbf{J}}$ ) are the WZW field and current which bosonize the upper (lower) spin-chains of the ladder. The initial values of the different coupling constants appearing in (13) are given by

$$
\lambda_{1}<0, \lambda_{2}=\lambda_{3}=J_{b}, \lambda_{4}=0
$$

In the bosonized representation the translation of chain by one site is equivalent to the discrete symmetry $g \rightarrow-g$. Therefore the operator $T_{\ell}$ is realized in the WZW model by the map,

$$
T_{\ell}:(g, \hat{g}) \rightarrow(-g, \hat{g})
$$

implying that $T_{\ell}$ is equivalent to the following change of couplings,

$$
\begin{array}{ccc}
J_{a} & \stackrel{T_{f}}{\rightarrow} & J_{a} \\
J_{b} & \stackrel{T_{f}}{\rightarrow} & -J_{b}
\end{array}
$$

Eq.(16) illustrates the relations $T_{\ell}|R V B\rangle=|H A L\rangle$ and $T_{\ell}|H A L\rangle=|R V B\rangle$, which stablish the equivalence between the RVB and Haldape states in the weak coupling limit. White has observed that the spins located in diagonal positions of the 2-ladder tend to form effective spins 1 , and using, what we call the $T_{\ell}$-transformation, he shows the equivalence between the two phases. This is done in 8 by introducing diagonal couplings in order to connect continously the $|R V B\rangle$ and $|H A L\rangle$ states. What we show in this letter is that this connection can be also thought as a discrete modular transformation by which the properties of both models can be put in one to one correspondence.

If $J_{a}<0$ both legs are in a ferromagnetic state with total spin $S_{\text {tot }}=N / 2$. A weak antiferromagnetic coupling, $J_{b}>0$, splits this degeneracy giving a state which, to first order in perturbation theory, is given by the singlet appearing in the Clebsch-Gordan decomposition $S_{\text {tot }} \times S_{\text {tot }}$. Obviously, the latter state is invariant under a shift of one of the legs. Thus the state $|M I X\rangle$, in the weak coupling regime, is invariant under $T_{\ell}$-duality, according to table 3.

The Strong Coupling Regime: $U_{\ell^{-}}$duality

In the strong coupling regime (i.e. $\left|J_{a} / J_{b}\right| \ll 1$ ) the rung Hamiltonian yields the zero order approximation, while the leg Hamiltonian acts as a perturbation.

The rungs, in a $A F$ ladder, are mostly in the spin 1 state which couple antiferromagnetically along the leg direction, yielding effectively a Haldane chain. The $U_{\ell}$ transformation, which simply permutes the two spins on the even rungs, leaves invariant the corresponding Haldane state (i.e. $U_{\ell}|H A L\rangle=|H A L\rangle$ ).

Next we shall show using perturbation theory that the $R V B$ and $M I X$ states are exchanged by $U_{\ell}$-duality.

For $J_{b}>>1$ the rungs are in a singlet state. The g.s. energy computed to $2^{\text {nd }}$ order in $J_{a}$ is given by 11 ,

$$
E_{0} / N=-\frac{3}{4} J_{b}-\frac{3}{8} \frac{J_{a}^{2}}{J_{b}}
$$

The first excited states form a band of spin 1 magnons,

$$
|k\rangle=\frac{1}{\sqrt{N}} \sum_{x=1}^{N} \mathrm{e}^{i k x}|x\rangle
$$

where $|x\rangle$ denotes the state with singlets on all rungs except at the position $x$ where it is a triplet. The dispersion relation $\omega(k)$ of $(18)$ is given, to $2^{\text {nd }}$ order in $J_{a}$, by 11 ,

$$
\omega(k)=J_{b}+J_{a} \cos k-\frac{1}{4} \frac{J_{a}^{2}}{J_{b}}(3-\cos 2 k)
$$


The action of $U_{\ell}$ on the magnons (18) is,

$$
U_{\ell}|k\rangle=|k+\pi\rangle
$$

Hence the spectrum of the $R V B$ and $M I X$ states, up to second order in $J_{a}$, are exchanged under $U_{\ell^{-}}$duality, as can be seen from the following identities satisfied by (17) and (19),

$$
\begin{gathered}
E_{0}\left(J_{a}, J_{b}\right)=E_{0}\left(-J_{a}, J_{b}\right) \\
\omega\left(k, J_{a}, J_{b}\right)=\omega\left(k+\pi,-J_{a}, J_{b}\right)
\end{gathered}
$$

The Intermediate Coupling Regime: $S_{\ell}$-duality When $\left|J_{a} / J_{b}\right| \sim 1$, the effective theory can be obtained by mapping the ladder into the non linear sigma model (NLSM)12.13,44. The values of the NLSM coupling constants are given in table 4 ,

\begin{tabular}{|c|c|c|c|}
\hline & $A A$ & $A F$ & $F A$ \\
\hline$\theta$ & 0 & $4 \pi S$ & 0 \\
\hline$g$ & $\frac{1}{S}\left(1+\frac{J_{b}}{2 J_{a}}\right)^{1 / 2}$ & $\frac{1}{S}$ & $\frac{1}{S}\left(\frac{J_{b}}{2\left|J_{a}\right|}\right)^{1 / 2}$ \\
\hline
\end{tabular}

Table 4: Parameters of the 2-ladder with spin $S$.

From these eqs. we get the curious relation,

$$
g_{A A}^{2}=g_{A F}^{2}+g_{F A}^{2}
$$

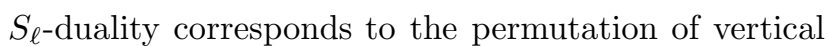
and horizontal bonds. Since on a 2-ladder there are twice as much horizontal bonds than vertical ones we expect a perfect balance between both couplings whenever $2\left|J_{a}\right|=$ $\left|J_{b}\right|$. In this case $g_{A F}=g_{F A}=g_{A A} / \sqrt{2}$. The change of $\theta$ by $4 \pi S$, when going from $F A$ to $A F$, does not affect the physics of the problem and recalls what happens with duality transformation in field theoriest.

Extrapolating the NLSM-map away the intermediate couplings we still find an agreement with table 3 . In the strong coupling regime both $g_{A A}$ and $g_{F A}$ go to the same asymptotical value, which agrees with the fact that the $U_{\ell}$ operation maps one ground state into the other. On the other hand, the value $g_{A F}=1 / S=2$ corresponds to the NSLM coupling of a spin chain with spin 1.

In the weak coupling regime the NLSM map is not reliable, however we see from table 4 , that in that limit $g_{A A}=g_{A F} \gg g_{F A}$ which agrees with the fact that $T_{\ell}$ interchages the $R V B$ and $H A L$ states. Of course in this limit we have two weakly coupled chains, which should be treated with bosonization techniques.

Summarizing our results we can say that the Haldane and the mixed phases are $S_{\ell}$-dual, while the RVB phase is self-dual under a $S_{\ell}$-transformation.

\section{Bond Moving Dualities}

What is the origin of the duality properties of ladders? In Conformal Field Theory or String Theory duality is an expression of modular invariance. Something of this sort exists also for spin systems. To show this we shall use a generalization of the Migdal-Kadanoff transformations, which consist in the substitution of couplings between NN sites by other NN or NNN couplings 6 . This is achieved by adding a potential $V$ to the Hamiltonian $H$, so that the new Hamiltonian $H^{\prime}=H+V$ has a g. s. energy ( and free energy) $E^{\prime}$ smaller than the g.s. energy $E$ of $H$, provided $\langle K\rangle=0$, where the v.e.v is taken respect to the g.s. of $H \mathrm{G}$. From fig. 1 we observe that the $T_{\ell}$-transformation corresponds to the bond moving potential,

$$
V_{T_{\ell}}=\sum_{n}\left(J_{b}^{\prime} \mathbf{S}_{1}(n) \cdot \mathbf{S}_{2}(n+1)-J_{b} \mathbf{S}_{1}(n) \cdot \mathbf{S}_{2}(n)\right)
$$

The Hamiltonian obtained adding (23) to the ladder Hamiltonian $H\left(J_{a}, J_{b}\right)$, is a new Hamiltonian $H\left(J_{a}^{\prime}, J_{b}^{\prime}\right)$, where $J_{a, b}$ are given by

$$
\begin{array}{ll}
J_{a}^{\prime} & =\quad J_{a} \\
J_{b}^{\prime} & =J_{b}\left\langle\mathbf{S}_{1}(n) \cdot \mathbf{S}_{2}(n)\right\rangle /\left\langle\mathbf{S}_{1}(n) \cdot \mathbf{S}_{2}(n+1)\right\rangle
\end{array}
$$

The expectation values in (24) are computed with respect to the g.s. of $H\left(J_{a}, J_{b}\right)$.Eq. (24) implies $\operatorname{sign}\left(J_{b}^{\prime}\right)=$ $-\epsilon_{a} \epsilon_{b}$, which indeed corresponds to a $T_{\ell}$-transformation.

Similarly the bond moving transformation which corresponds to $U_{\ell}$ gives,

$$
\begin{aligned}
& J_{b}^{\prime}=\quad J_{b} \\
& J_{a}^{\prime}=J_{a}\left\langle\mathbf{S}_{1}(n) \cdot \mathbf{S}_{1}(n+1)\right\rangle /\left\langle\mathbf{S}_{1}(n) \cdot \mathbf{S}_{2}(n+1)\right\rangle
\end{aligned}
$$

Finally the $S_{\ell}$-tranformation can be derived from its definition $S_{\ell}=T_{\ell} U_{\ell} T_{\ell}$. For all these transformations, duality would amount to the equality $E\left(J_{a}, J_{b}\right)=$ $E\left(J^{\prime}{ }_{a}, J^{\prime}{ }_{b}\right)$. The variational principle underlying the Migdal-Kadanoff transformation only guarantees that $E\left(J_{a}, J_{b}\right)>E\left(J_{a}^{\prime}, J_{b}^{\prime}\right)$, however after the results obtained above using perturbative and field theoretical methods, we have good reasons to believe that the replacement of inequalities by equalities for the energies and free energies yields a good approximation. Further studies are necessary to fully stablish these facts.

Beyond the 2-ladder Most of the results shown so far are generalizable to the case of even-ladders with periodic $\mathrm{BC}$ 's along the rungs. The $T_{\ell}$-transformation is given by the shift of one lattice space of the even legs respect to the odd legs, so that the rungs become zigzag lines across the ladder. In fact, this definition applies to all types of ladders, even and odd, with different BC's across the rungs. The $U_{\ell}$ transformation consists in the shift by one lattice space of the even rungs, so that the legs become zig-zag lines along the ladder. The new coupling constants, obtained upon these transformations, are also given by the eqs.(24) and (25).

For odd-ladders with open BC's along the rungs, there seems to be not a sensible definition of the $U_{\ell}$ and $S_{\ell}$ transformations, as we did above for $T_{\ell}$. The odd-ladders of type $A A$ are in the same universality class as the spin $1 / 2$ antiferromagnetic Heisenberg chain, whose g.s. we 
shall denote as $\left|A_{1 / 2}\right\rangle$. On the other hand the $n_{\ell}$-ladders ( $n_{\ell}$ : odd) with $A F$ couplings are, at least in the strong coupling regime, equivalent to spin $n_{l} / 2$ antiferromagnetic chain, whose g.s. we shall denote as $\left|A_{n_{\ell} / 2}\right\rangle$. The role of $T_{\ell}$ is to exchange the $A A$ and $A F$ couplings, which implies that $T_{\ell}\left|A_{1 / 2}\right\rangle=\left|A_{n_{\ell} / 2}\right\rangle$. The previous equivalence can be stablished for every regime of couplings using, as we did for the 2-ladder, the appropiated technique. Thus for intermediate couplings, where we can use the mapping of the ladder into the NLSM $B$, we get the parameters $\theta_{A A}=\pi$ and $\theta_{A F}=\pi n_{\ell}$, which coincide modulo $2 \pi$. The odd-ladders with $F A$ and $F F$ couplings are equivalent to ferromagnetic Heisenberg chains with spins $1 / 2$ and $n_{\ell} / 2$ respectively. Their g.s. is invariant under $T_{\ell}$.

In summary we have seen that the even and odd ladders have quite different duality properties which is of course a manifestation of the fact that they both belong to different universality classes.

Acknowledgements: We would like to thank R. Shankar, E. Fradkin, L. Balents and G. Mussardo for useful conversations. Work partially supported by the grants NSF PHY94-07194 and the DGES (G.S.) and by CICYT under contract AEN93-0776 (M.A.M.-D.).

* On leave of absence from Instituto de Matemáticas y Física Fundamental, C.S.I.C., Serrano 123, 28006-Madrid, Spain.

${ }^{1}$ N. Seiberg and E. Witten, Nucl. Phys. B 426, 19 (1994).

${ }^{2}$ J. Polchinsky, TASI Lectures.

${ }^{3}$ C. Itzykson and J.M. Drouffe, "Statistical Field Theory", CUP (1991).

${ }^{4}$ E. Dagotto and T.M. Rice, Science 271, 618 (1996).

${ }^{5}$ P. Ginsparg, Les Houches Lecture Notes 1988 on "Fields, Strings and Critical Phenomena".

${ }^{6}$ L.P. Kadanoff, Ann. Phys. (NY) 100, 359 (1976); A. A. Migdal, Sov. Phys. JETP 42, 413 and 743 (1976).

${ }^{7}$ K. Hida, Phys. Rev. B 45, 2207 (1992)

${ }^{8}$ S. R. White, Phys. Rev. B 53, 52 (1996).

${ }^{9}$ F.D.M. Haldane and I. Affleck, Phys. Rev. B 36, 5291 (1987).

${ }^{10}$ K. Totsuka and M. Suzuki, J. Phys.: Conds. Matt. 7, 6079 (1995).

${ }^{11}$ M. Reigrotzki, H. Tsunetsugu and T. M. Rice, J. Phys.: Conds. Matter 6, 9235 (1994).

${ }^{12}$ D. Senechal, Phys. Rev. B 52, 15319 (1995).

13 G. Sierra, J. Math. Phys. A 29, 3299 (1996).

14 A.K. Kolezhuk and H.J. Mikeska, Phys. Rev. B 53, 8848 (1996). 


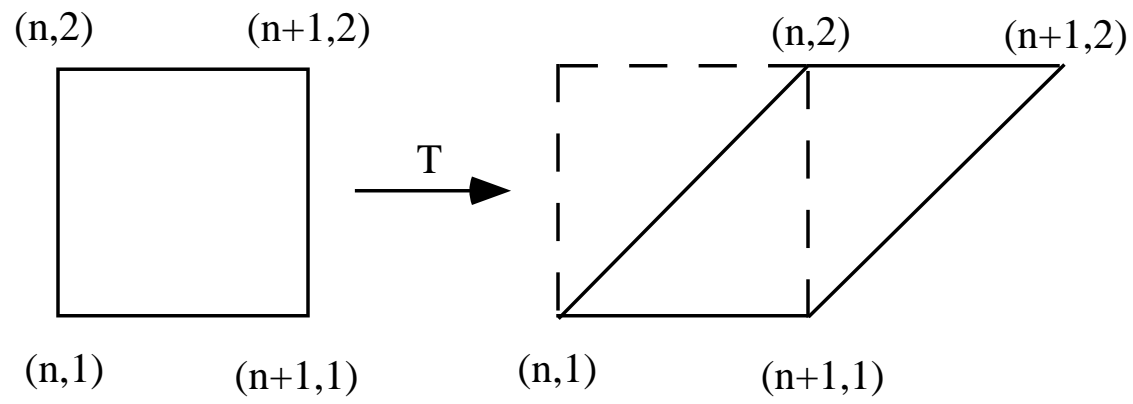

Figure 1 


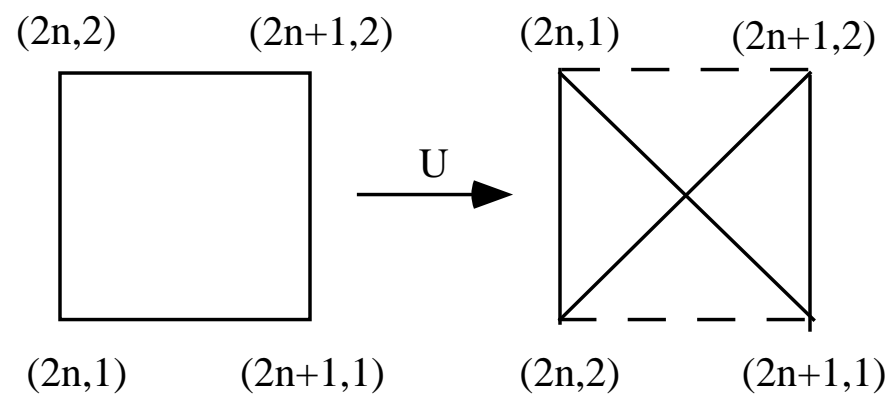

Figure 2 